\title{
3 Der, „State of the Art" der sozialwissenschaftlichen Forschung zum Interaktionsfeld der Wohnnachbarschaft im urbanen Kontext
}

\subsection{Der Wohnbereich und die Nachbarschaft als sozialer Interaktionskontext in urbanen Agglomerationen}

Wir verstehen in der vorliegenden Studie unter Nachbarschaft eine sozialwissenschaftliche Bezeichnung für räumliche Wohn- und Siedlungsnähe sowie für die aus dieser Voraussetzung resultierenden sozialen Beziehungen spezifischer Art.

Das soziale Wohnumfeld wird durch die soziale und ethnische Zusammensetzung der Nachbarschaft konstituiert. Die Nachbarschaft ,eine soziale Gruppe, deren Mitglieder primär wegen der Gemeinsamkeit des Wohnortes miteinander interagieren " (Pfeil 1972: 267), stellt eine Erweiterung des Wohnbereichs dar und besitzt ein erhebliches Interaktionspotential, wobei die eigentliche Nachbarschaftsgruppe klein ist und in der Regel nur wenige Haushalte umfasst. Nachbarschaft beinhaltet zwei Elemente: die räumliche Nähe der Wohnstandorte und die sozialen Interaktionen (Grafmeyer 1994; Hise 2003: 4). Nachbarschaft weist also eine doppelte Bedingtheit auf: durch soziale und physisch-geographische Gegebenheiten.

Großstädtische Nachbarschaft bildet keine dauerhafte, konsistente Gemeinschaft und wird nicht durch ein normatives Gefüge des Zusammenlebens konstituiert bzw. zusammengehalten, großstädtische Nachbarschaft reduziert sich auf die Gemeinsamkeit des Wohnortes und damit auf einen räumlichen Kontext, in dessen Rahmen sich erst die Möglichkeit und die Chance für einen unmittelbaren Sozialkontakt ergibt. Die lokal bedingten, sozialen Beziehungen basieren auf dem Nebeneinanderwohnen. „Nachbarschaft ist nicht personal, sondern lokal zu sehen" (Auer 1978: 109). Die räumliche Nähe ist zwar eine notwendige Voraussetzung, aber keine hinreichende Bedingung für soziale Interaktionen. ${ }^{11}$ Die Nähe der Wohnstandorte wird als gruppenbildendes Merkmal verstanden. Die Nachbarn werden dadurch von den Verwandten oder Freunden unterschieden, die zwar auch in räumlicher Nachbarschaft wohnen können, welche aber nicht die Voraussetzung für diese Kontakte darstellt.

Die Wohnnachbarschaft besitzt zwar ein erhebliches Interaktionspotential, impliziert aber stets die Interaktionen von weniger Individuen miteinander, als potentielle Nachbarschaftsbeziehungen aufgenommen werden könnten. Räumliche Nähe ist zwar eine notwendige Voraussetzung, aber keine hinreichende Bedingung für soziale Interaktionen. Räumliche Nähe ist auch nicht automatisch mit intensiven Binneninteraktionen einer sozialen Gruppe gleichzusetzen. Zwar kann die räumliche Nähe einer-

11 Es sei hierbei auf den Unterschied zwischen ,,geographical proximity“ und „organized proximity " verwiesen (Rallet \& Torre o. J.). 
seits auf eine erhöhte Interaktion zwischen den Mitgliedern einer ethnischen Gruppe auf einer Ebene hinweisen, andererseits spielt die räumliche Nähe keineswegs bei allen Netzwerken die wichtigste Rolle. Sie ist zwar eine notwendige Voraussetzung, aber keine hinreichende Bedingung für soziale Interaktionen.

„Nachbarschaft“" wurde vor allem in der Stadtplanung der 1970er Jahre zu einem ideologiekritisch gebrauchten Zentralbegriff (vgl. Hamm 1973). Zu den „Übeln“ des urbanen Wohnens wurde der ,,Verlust von Heimat “ in Vierteln mit hoher Mobilität und kurzer Wohndauer, die Unverbindlichkeit und Anonymität der sozialen Beziehungen in der Großstadt sowie die Reduktion oder gar der allgemeine Verlust nachbarschaftlicher Beziehungen gezählt. ${ }^{12}$ Klassische Stadtsoziologen wie Wirth und Park, aber auch Greverus et al. (1994) und (Rogers \& Vertovec 1995: 17) charakterisieren urbane Beziehungen nach folgenden Merkmalen: ,low intensity, displaying cynism, anomic attitudes or lack of attachment ".

Städtische und dörfliche Nachbarschaft wurden in stadtplanerischen Publikationen der 1970er Jahre einander antithetisch gegenübergestellt, wobei letztere über Gebühr idealisiert wurde. Die Nachbarschaftsbeziehungen im ländlichen Milieu überlappen einander eher als die in der Stadt, wo jede Person in unterschiedlichen Kontexten mit unterschiedlichen Personen soziale Beziehungen unterhält (Rogers \& Vertovec 1995: 17; Kreiser 1997: 72). Das heißt, soziale Beziehungen im ländlichen Milieu sind vielfältiger (vgl. die ,,multiplexity“ von sozialen Beziehungen nach Mitchell 1987) als im urbanen Raum.

Dass der Verlust des rigiden „Nachbarschaftskorsetts“ in der Großstadt dem Individuum auch erheblich größere Freiräume und Unabhängigkeit von sozialer Kontrolle verschafft, fand kaum Berücksichtigung. Diese Orientierung ging einerseits auf eine kulturpessimistische Interpretation von Urbanisierung im allgemeinen zurück (großstädtisches Leben wurde mit Verlust von Gemeinschaft, Werteverfall und sozialen Desorganisationserscheinungen assoziiert), andererseits wirkten Einflüsse der amerikanischen Stadtforschung, in erster Linie der Sozialökologie, die von einer „Naturgesetzlichkeit“ der „,community“-Bildung als grundlegender Form der sozialräumlichen Gliederung von Großstädten ausging (vgl. Friedrichs 1977).

Die europäische Stadtplanung heftete nicht nur das Nachbarschaftskonzept auf ihre Fahnen, sondern auch das „Credo der sozialen Mischung“ (Häussermann 1995: 14). Konträr zur Sozialökologie wurden kulturelle und soziale Unterschiede weitgehend negiert. Die stadtplanerisch konzipierten Nachbarschaften, waren in der Folge meist zu heterogen, um wirklich funktionieren zu können.

12 Innerhalb des urbanen Kontextes bestehen hinsichtlich der Intensität von Nachbarschaftsbeziehungen erhebliche Unterschiede zwischen Alt- und Neubauvierteln. Neubaubewohner haben häufiger ein distanzierteres bzw. anonymeres Verhältnis zu ihren Nachbarn als Althausbewohner (Schulz zur Wiesch 1982: 267). 


\subsection{Die räumliche Abgrenzung von Wohnnachbarschaft}

Die Nachbarschaft repräsentiert eine räumlich-soziale Einheit auf einer sozialen Mikroebene, in der sich durch den Zuzug von Migranten Abstoßungsreaktionen seitens der „etablierten“ Nachbarn herausbilden können. Es stellt sich allerdings die Frage, wo die Grenzen der „Nachbarschaft“ als Untersuchungseinheit zu ziehen sind und dies in strikt räumlicher Hinsicht. Das weitere Kommunikationsfeld des Wohnhauses wird durch die Baueinheit Aufgang in sozial relativ geschlossene Zonen segmentiert.

Eichener (1988: 121) postuliert einen Zusammenhang zwischen der Kleinheit räumlicher Einheiten und einer steigenden Relevanz derselben als Nachbarschaftseinheit im Bewusstsein und Handeln ihrer Bewohner. Räumlichen Einheiten auf höheren Ebenen weist er nur dann Handlungswirksamkeit zu, wenn diese als räumlichsoziale Einheiten wahrgenommen werden. Diese Wahrnehmung ist abhängig von:

- der räumlichen Geschlossenheit: Je homogener die Baustruktur und städtebauliche Gestaltung eines Gebietes ist und je deutlicher es sich nach außen abgrenzt, desto stärker ist es als räumliche Einheit identifizierbar.

- der Überschaubarkeit: Überschaubarkeit fördert die subjektive Wahrnehmung als eine Einheit.

- der Funktion als Interaktionsfeld: Je stärker durch die gemeinsame Nutzung von infrastrukturellen Einrichtungen Interaktionen zwischen den Bewohnern ermöglicht werden, desto größer ist die Wahrscheinlichkeit, dass der Ort als Element im Handeln sozial wirksam wird.

- die Zusammengehörigkeit: die Identifikation von Individuen mit ihrer räumlichen Wohnumgebung (Haus, Straße, Siedlung, Wohnviertel) ist umso stärker, je homogener die Nachbarschaft hinsichtlich verbindender Merkmale ist (Sozialstatus, Wertvorstellungen, Lebensgewohnheiten).

Die Grenze zwischen privater und öffentlicher Sphäre im Kontext des Wohnens bildet üblicherweise die Wohnungstüre. In den gründerzeitlichen Bassenahäusern mit Wasser und WC auf dem Gang wird ein Teil der privaten Sphäre in die Halböffentlichkeit der Stiegenhäuser verlagert. Dies ist ein gutes Beispiel dafür, wie architektonische Elemente Rückwirkungen auf das Verhalten ihrer Bewohner besitzen. Es kommt somit zu einer Verschiebung der Grenzen zwischen Öffentlichkeit und Privatheit. Die halböffentlichen nachbarlichen Kontaktzonen besitzen eine Mittelstellung zwischen öffentlichem und privatem Raum (Bahrdt 1961; Herlyn 1970: 168).

Die Komponente des räumlichen Distanzfaktors ist auf der Ebene des Wohnhauses durch zwei Dimensionen repräsentiert:

- die vertikale Dimension (das Stockwerk),

- die horizontale Dimension (Aufgänge bzw. Stiegen).

Innerhalb dieser beiden Dimensionen wirken baulich-strukturelle Merkmale und Besonderheiten (Aufzug, Balkone und Terrassen, Gestaltung der Stiegenhäuser, GangWC etc.), die Kontaktchancen eröffnen, fördern oder behindern. Die Wohnform de- 
terminiert zwar in einem gewissen Ausmaß die soziale Kontaktbereitschaft, jedoch wirkt von der Wohnform aufgezwungene Ballung nach Thum (1979: 123) keineswegs ausschließlich kontaktfördernd. Die höchste Bereitschaft zu Sozialkontakten wird bei Bewohnern aufgelockerter Bauformen postuliert. Bestehen auf engem Raum heterogene Wohnformen nebeneinander, so werden ihre positiven und negativen Charakteristika im Erleben der Bewohner verstärkt, woraus eine Tendenz zur Abkapselung innerhalb der eigenen Bezugsgruppe resultiert.

Die Entfernung, d.h. der Distanzfaktor, sowie die relative Geschlossenheit von baulich-strukturellen Einheiten schaffen innerhalb baulicher Einheiten Kontaktgelegenheiten und behindern sie nach außen, da die Bewohner entfernter liegender Wohneinheiten weniger Gelegenheiten zu Begegnungen finden. Es existiert eine konsistente Beziehung zwischen der Wahl der Kontaktpartner und der räumlichen Distanz. In der Regel wird das eigene Treppenhaus von den Hausbewohnern als eine wichtige räumliche Einheit wahrgenommen, dem eigenen Stockwerk kommt nach dem Kriterium der räumlichen Nähe eine noch größere Bedeutung zu (Gronemeyer \& Bahr 1977: 150 f.). Räumliche Kontaktfelder finden sich also im Treppenhaus und auf der Ebene des Stockwerkes.

Die Wohnnachbarschaft stellt die Arena der zufälligen Begegnungen dar. Bereits frühe Arbeiten zur städtischen Nachbarschaft (z. B. McClenahan 1929: 30 ff.) wiesen auf das Faktum hin, dass ,zufällige“ Kontakte durch das Zusammentreffen im Stiegenhaus oder Hof sowohl intensivere Interaktionen fördern, genauso aber auch zu verstärkten Konflikten führen können, wenn die Verhaltensstandards der Interaktionspartner zu sehr divergieren. Bestehen auf Seiten beider Interaktionspartner Motivationen für Interaktionen, so können daraus positive Nachbarschaftsbeziehungen resultieren. Diese sind im Kontext ethnisch gemischten Wohnens schon deshalb so wichtig, da Sozialkontakte ein ebenso einfaches wie wirkungsvolles Mittel gegen Fremdenfeindlichkeit (Kohlbacher \& Reeger 1999a) sind und ,, der Wohnbereich eine gute, fast ideale Gelegenheit zu verständnisfördernder Begegnung “ (Ludl 2003: 19) bietet. ${ }^{13}$

Eichener (1988: 151) plädiert, ausgehend von deutschen Verhältnissen, dafür, die Straße als Nachbarschaftseinheit anzusehen. Die Straße als Bezugseinheit erschien uns aber für eine Untersuchung der Wiener Situation, vor allem im Geschosswohnbau, als räumlich zu weit gefasst. Bereits der alltägliche Wiener Sprachgebrauch belegt dies: Wer in einem Wiener Wohnhaus von ,seinen Nachbarn“ spricht, meint im engeren Sinn die Wohnparteien im eigenen Stockwerk, im weiteren Sinn die Bewohner auf der eigenen Stiege, eventuell - aber nur unter der Einschränkung, dass es sich um ein Haus mit relativ wenigen Parteien und einer übersichtlichen Bewohnerstruktur han-

13 Die Erfahrungen von Verwaltungen gemeinnütziger Bauträger sprechen dafür, dass sich nachbarschaftliche Konflikte durch verstärkte soziale Kontakte der Bewohner in viel höherem Ausmaß von selbst und ohne Intervention der Hausverwaltungen lösen lassen (Ludl 2003: 20). 
delt - die Bewohner des ganzen Hauses. In Bezug auf Besuchskontakte ist das eigene Stockwerk primäres Kommunikationsfeld, als Kommunikationszone ersten Ranges fungiert primär die eigene Stiege. Etwas anders ist die Situation in den Bezirksteilen mit lockerer Einfamilienhausbebauung. Hier gilt, was auch in kleineren Gemeinden in der Regel der Fall ist, dass die Nachbarschaft großräumigere Dimensionen annimmt und sich nicht nur auf die umliegenden Häuser, sondern auch auf die ganze Straße beziehen kann. Die relevante Nachbarschaftseinheit im kleinsten Umfang stellt also das eigene Stockwerk dar, im etwas weiteren Rahmen die Stiege bzw. das Wohnhaus.

Da Anwesenheit und Verhalten von Nachbarn mit Migrationshintergrund umso direkter empfunden werden, je näher diese im Verbund mit Österreichern wohnen, sind nach unserem Dafürhalten Stiege und Wohnhaus jene räumlichen Kontexte, in deren Rahmen Kontakte am ehesten stattfinden.

Dem Face-to-face-Kontakt im unmittelbaren Wohnumfeld, mag er in modernen Großstädten auch noch so distanziert und anonymisiert erfolgen, ist die Rolle eines sozialen Relais zuzuschreiben. Alleine schon die geringe physische Distanz bedingt die Ausbildung einer anderen Qualität von Sozialbeziehungen im Kontext der Nachbarschaft als etwa bei Kontakten im öffentlichen Raum von Parks oder Straßen (Klages 1968; Hamm 1973; Auer 1978; Schulz 1978; Engelhard 1986; Rigger 1988).

\subsection{Nachbarschaftsforschung: historischer Abriss, Grundfragen und empirische Befunde}

Ausgehend von dem von Howard (1904) entwickelten und in den 1920er Jahren praktisch umgesetzten Modell der „neighbourhood-units“ boomte die Nachbarschaftsforschung vor allem in den USA der 1950er und 1960er Jahre, wobei hier als ältere „Klassiker“ "nur die Arbeiten von Caplow und Forman (1950) sowie von Festinger et al. (1959) angeführt werden sollen. Ernest W. Burgess und Robert Park von der "Chicago School of Social Ecology“" propagierten die Annahme, dass die Face-toface-Beziehungen im nachbarschaftlichen Kontext eine Art von gemeinschaftlichem Zusammenhalt kreieren würden, der einen Gegensatz zu den hohen Levels von Anomie und Entwurzelung in der industriellen Stadt bilden könnte.

Vor allem in den 1960er und 1970er Jahren fand die sozialwissenschaftliche Auseinandersetzung mit der Wohnnachbarschaft auch im deutschen Sprachraum Eingang (vgl. Bahrdt 1960, 1968, 1974; Gans 1961, 1974; Herlyn 1970; Pfeil 1972; Vierecke 1972; Hamm 1973) bei wieder abnehmender Bedeutung in den 1980er Jahren (Langkau-Herrmann 1981; Engelhard 1986). Randlich haben sich auch Affeld (1974), Bahrdt (1968) und Gans $(1962,1974)$, deren Hauptforschungssujet die Untersuchung von städtebaulichen Einheiten auf ihre Homogenität bzw. Heterogenität hin war, mit der Wohnnachbarschaft auseinandergesetzt. Weitere Studien wurden von EMNID (1982), Dietl (1993) und Schubert (1990) durchgeführt. Letzterer hat das Solidaritätspotential der Wohnnachbarschaft untersucht. 
Das Phänomen der ethnisch gemischten Nachbarschaft ist in Deutschland und in Österreich weitgehend gleichzeitig und in größerem Umfang erst im Laufe der 1970er Jahre aufgetreten, als die „Gastarbeiter“ zunehmend aus ihrer Wohnseklusion in firmeneigenen Massenquartieren heraustraten und durch Familiennachzug zu Wohnnachbarn von Inländern wurden. Vor allem unter den Aspekten der Störungswahrnehmung und der Vorurteilsbildung widmeten sich u. a. Hemmer \& Leminsky (1974), Becher \& Erpenbeck (1977), Hoffmeyer-Zlotnik (1977), Elschenbroich \& Schweitzer (1982), Hill (1984), Gehring \& Böltken (1985), König et al. (1986), Eichener (1988) sowie Böltken $(1991,1994)$ der Situation von Zuwanderern im Wohnbereich und somit auch Aspekten der interethnischen Wohnnachbarschaft, die aber zumeist nicht im Fokus der angeführten Untersuchungen stand.

Der „state of the art" des aktuellen Forschungsstands dokumentiert die Wichtigkeit des Interaktionsfeldes der Wohnnachbarschaft für interethnische Begegnungen (vgl. dazu Becher \& Erpenbeck 1977). König et al. (1986) schrieben dem Wohnbereich sogar die Funktion des wichtigsten Kontaktfeldes zwischen Inländern und Migranten zu. Das unmittelbare Wohnumfeld bietet nach ihrer Einschätzung insbesondere Immigrantinnen die Chance, informelle Kontakte zu Einheimischen herzustellen. Becher \& Erpenbeck (1977) unterstrichen die Rolle des Wohnbereichs für die Etablierung interethnischer Freundschaften. Hoffmeyer-Zlotnik (1977) hat die Bedeutung einer höheren Kontaktfrequenz zwischen In- und Ausländern im Wohnbereich vor allem im Hinblick auf ihre vorurteilsreduzierende Wirkung analysiert. Schulz zur Wiesch (1982) identifizierte die Alltagswohnerfahrungen als Hauptdeterminante der Bereitschaft zu einem konfliktfreien Zusammenleben zwischen in- und ausländischer Wohnbevölkerung. Bonacker und Häufele (1986) arbeiteten eine erhebliche Bandbreite der Intensität und Frequenz interethnischer nachbarschaftlicher Kontakte heraus, wobei im urbanen Bereich soziale Distanz jedoch das vorherrschende Muster darstellt.

Distanz gegenüber dem Nachbarn stellt einen soziokulturell bedingten Verhaltensstandard dar (Barre et al. 1977: 148). In einer Reihe von Studien wurde der Einfluss interethischer Nachbarschaftskontakte auf Einstellungen gegenüber Zuwanderern untersucht. Gehring \& Böltken (1985), die sich mit der Kontaktproblematik besonders intensiv auseinandersetzten, weisen den nachbarschaftlichen Kontakten bloß eine inferiore Rolle gegenüber den intensiveren Freundschafts- und familiären Kontakten zu. Böltken (1991, 1994), der sich explizit dem Komplex der interethnischen Nachbarschaftsbeziehungen widmete, ortete im Bereich der Wohnnachbarschaft vor allem im Umgang mit türkischen Zuwanderern ein manifestes Problem der deutschen Wohnbevölkerung.

Für Österreich lagen zunächst nur wenige Forschungsbefunde vor (Schulz 1978). Habinski (1992), Riedl (1992) und Nachbagauer (1992) haben in ihren Diplomarbeiten interethnische Nachbarschaftskontakte als einen Teilaspekt berücksichtigt, diese jedoch nur auf Basis narrativer Interviews und sehr kleiner Samples untersucht. Sie stimmten in dem Resultat überein, dass interethnische Nachbarschaftskontakte in Wien in verschiedenen Varianten zwar vorhanden sind, meist aber in distanzierter 
Form ablaufen. Die Studie der GSD-Gesellschaft für Stadt- und Dorferneuerung (Reppé \& Reiter 1997) basierte auf einem großen Sample von qualitativen Interviews in sanierten Wiener Gemeindewohnbauten. Ausschließlich aus der Perspektive der „Altösterreicher" hat Kohlbacher (2000) das ethnisch gemischte nachbarschaftliche Zusammenleben untersucht. Als einen Teilaspekt von Xenophobieerfahrungen und basierend auf den Daten einer Ausländerbefragung haben Kohlbacher \& Reeger (2003b) auch den Wohnbereich berücksichtigt. Dem Wohnmodell „Inter-ethnische Nachbarschaft" in all seinen Facetten hat sich eine Reihe von Autoren im Kontext eines reichhaltigen Sammelbandes gewidmet (Ludl 2003).

Weiters sei hier auf die Dokumentation zur Vortragsveranstaltung „Gemeindebau im Gespräch - Entwicklungen und Perspektiven im kommunalen Wohnbau in Wien “14 verwiesen, die neueste und zum größeren Teil noch nicht publizierte Studien zum interethnischen Zusammenleben in Wiener Gemeindebauten beinhaltet: So etwa die Untersuchung der Studenten des Forschungslabors „Angewandte Stadtforschung“ am Institut für Soziologie der Universität Wien mit dem Titel „Zusammenleben im Gemeindebau: Konflikte und Potenziale“, weiters die Erhebungen des Instituts für Kultur- und Sozialanthropologie (Proseminar „Qualitative Methoden“, Leitung: Maria-Anna SixHohenbalken ), die von März bis Juni 2005 in verschiedenen Gemeindebauten in Floridsdorf durchgeführt wurden (,BewohnerInnen mit Migrationshintergrund im Gemeindebau. Zwischen Fremdheit und Vertrautheit, Nähe und Distanz") und auf die Diplomarbeit (2006) von Rosmarie Nigg (Institut für Soziologie, Universität Wien) mit dem Titel „Soziale Körper in Transformation. Eine empirische Untersuchung über Integration von ZuwanderInnen und Nachbarschaft in Wiener Gemeindebauten". Im Karl-Marx-Hof hat Elisabeth Strasser (Institut für Kultur- und Sozialanthropologie, Universität Wien) von Mai 2005 bis Februar 2006 eine Befragung zum Thema „Nachbarschaft und soziale Netzwerke im Karl-Marx-Hof" im Rahmen des derzeit in acht europäischen Staaten durchgeführten EU-Projekts KASS („,Kinship And Social Security" - Verwandtschaft und soziale Sicherheit") durchgeführt. Die ethnographischen Erhebungen bestanden aus strukturierten genealogischen und qualitativen Interviews mit Bewohnern des Karl-Marx-Hofs, Experteninterviews mit Vertretern lokaler Einrichtungen, sowie „,eilnehmender Beobachtung“ im und um den Karl-MarxHof. Insgesamt wurden rund 150 Personen in die Untersuchung eingebunden.

Alle genannten Erhebungen haben Fragen der interethnischen Nachbarschaft auf der Grundlage nicht repräsentativer Samples mehr oder weniger detailliert untersucht und sind zu mitunter beträchtlich divergierenden Resultaten gelangt. Resümierend ist aus dem Gesagten also abzuleiten, dass die Resultate der im deutschen Sprachraum bislang geleisteten Studien hinsichtlich der Rolle und Qualität der interethnischen Wohnnachbarschaft äußerst heterogen, ja einander geradezu widersprechend sind.

14 Vgl. dazu auf der Homepage des Instituts für Soziologie der Universität Wien: http://www.gerda.univie.ac.at/ifs/files/workshop/gemeindebau.pdf?PHPSESSID=2b98a139 ed6c668394fcf8c52bd070c6. 


\subsection{Nachbarschaftliches Interaktionsverhalten}

Hamm (1973: 99 ff.) fomulierte die folgenden, auf das konkrete Nachbarschaftsverhalten bezogenen Thesen:

- Nachbarschaft ist eine der sozialen Bezugsgruppen, an deren Normen sich das Verhalten der Menschen orientiert.

- Die Gesamtzahl der Bezugsgruppen, denen ein Individuum außerhalb der Nachbarschaft angehört, entscheidet über die Bedeutung der Nachbarschaftsgruppe im Kontext der Verhaltensorientierung.

- Je weiter eine Gesellschaft arbeitsteilig organisiert ist, desto größer ist die Zahl der Bezugsgruppen.

- Die Zahl der Bezugsgruppen ist abhängig vom Einkommen, der Stellung im Lebenszyklus, der beruflichen Position und dem Lebensalter.

- Nachbarschaftliches Verhalten orientiert sich an Normen, denen unterschiedliche Verbindlichkeit zukommt.

- Die Nachbarschaftsgruppe kann Aufgaben der Nothilfe, der Kommunikation und der sozialen Kontrolle übernehmen.

- Die räumliche Nähe der Wohnungen ist das Kriterium für die Auswahl möglicher Interaktionspartner; über die faktische Interaktionsdichte entscheiden jedoch zusätzlich vorhandene Gemeinsamkeiten zwischen den Nachbarn (vgl. auch DemmlerMosetter 1982: 112 f.).

- Der Bezugsgruppe Nachbarschaft kommt je nach sozialen und ökonomischen Merkmalen individuell unterschiedliches Gewicht zu.

Die räumliche Ausdehnung nachbarlichen Verhaltens hängt von der ,emotionalen Ausdehnungsfähigkeit“ (Klages 1968: 72) des Individuums ab. Als fest umgrenzter Kernbereich des nachbarlichen Verhaltens ist nur das Haus selbst anzusehen (Auer 1978: 110). Hier sind jedoch das gegenseitige Distanzbedürfnis und die Konfliktmöglichkeiten am stärksten ausgeprägt. Die Beschaffenheit des Hauses, seine Form und architektonische Gliederung beeinflussen die , räumliche Konfiguration der nachbarlichen Kommunikation " (Auer 1978: 110). Heil (1969, 1971) hat bereits früh nachgewiesen, dass sich deutliche Unterschiede zwischen Alt- und Neubauvierteln hinsichtlich der räumlichen Konfigurationen nachbarschaftlichen Verhaltens und des daraus resultierenden Kontaktverhaltens feststellen lassen. In Altbauten ist der Typus eines nachbarschaftszentrierten Verhaltens eher zu finden. In Neubauten sind nachbarliche Interaktionen stärker auf das engste Wohnumfeld (Stockwerk) beschränkt.

Distanz gegenüber den Nachbarn stellt einen soziokulturell bedingten Verhaltensstandard im urbanen Kontext dar, wobei anzunehmen ist, dass hinter dem Distanzwunsch Konfliktangst steht, durch die latente Kommunikationsbedürfnisse verdeckt werden (Gronemeyer \& Bahr 1977: 148). Besuchskontakte im nachbarlichen Umfeld gelten als prekär und werden daher im sozialen Beziehungsfeld der Nachbarschaft eher nicht praktiziert. Im Gegensatz dazu basieren enge freundschaftliche Kontakte in 
der Regel auf dem Prinzip der freien Wahl der Kontaktpartner und stellen daher nicht durch räumliche Nähe vermittelte „Zwangskontakte“ dar.

Einige empirische Studien belegen die Bedeutung der räumlichen Distanz dahingehend, dass z.B. nachbarschaftliche Besuchskontakte sehr häufig auf das eigene Stockwerk eingegrenzt sind. Dies deutet darauf hin, dass das Prinzip von , Sympathie durch räumliche Nähe bzw. Kontakt" (Homans 1967, 1972; Gronemeyer \& Bahr 1977: 156) eine gewisse Wirksamkeit besitzt und sogar wichtiger sein kann als die freie Wahl der Bezugspersonen. Engere Nachbarschaftskontakte und Nebeneinanderwohnen bedingen einander demzufolge also geradezu.

Im Gegensatz dazu postuliert Weeber (1971: 122), dass räumliche Konfigurationen per se keinen allzu bedeutsamen direkten Einflussfaktor auf das Kommunikationsverhalten darstellen. Die entscheidende Variable dürfte seiner Ansicht nach die Wohndauer sein. Mit zunehmender Wohndauer in einem Haus tritt zunehmende soziale Integration seiner Bewohner ein. Die Frage nach der notwendigen Dauer dieses Anpassungsprozesses muss aufgrund fehlender Longitudinalstudien allerdings unbeantwortet bleiben (Schulz 1978: 31).

Erzwungenes Nachbarschaftsverhalten wird durch gemeinsam zu nutzende Einrichtungen (Gang-WC, Bassena, Waschküche, Trockenboden etc.) bedingt und besitzt den Charakter unvermeidlicher Kontakte. Eine selektive Beschränkung der Kontakte ist dabei weitgehend ausgeschlossen. Um die gewünschte soziale Distanz im erzwungenen Kontakt aufrechtzuerhalten, kommt es zu einer starken Verhaltensritualisierung. Im Gegensatz dazu ist das spontane Nachbarschaftsverhalten auf Geselligkeit oder Hilfestellung orientiert, greift tiefer in die private Sphäre ein, ist selektiv und zeitlich begrenzt. Im Allgemeinen bleibt aber der nachbarliche Kontakt auf die Involvierung oberflächlicher Schichten der eigenen Persönlichkeit beschränkt.

Alle Studien zu Nachbarschaftskontakten in Großstädten stimmen darin überein, dass nachbarliche Kontakte sich nur schwer systematisieren lassen. Sie erstrecken sich über ein Kontinuum von größtmöglicher Distanz und Anonymität bis zu engem freundschaftlichem Kontakt. ${ }^{15}$ Das Typische für den städtischen Kontext ist, dass einem die Nachbarn zwar (teilweise) bekannt, jedoch nicht freiwillig gewollt sind, daher kann nur ein gewisser sozialer Abstand garantieren, dass Konflikte und Reibungen das Zusammenleben nicht unerträglich gestalten.

15 Eine Erhebung in 392 Salzburger Haushalten (Rose 1975: 75) ergab, dass die beiden Extreme nachbarschaftlichen Verhaltens, die völlige Anonymität mit 1\% und der enge freundschaftliche Kontakt mit 5\% unter den Bewohnern von Hochhaussiedlungen eher als Ausnahmefälle zu bezeichnen sind. Es dominiert der Nachbarschaftstypus der ,reduzierten Distanz" (59\%). 


\subsection{Interethnische Wohnnachbarschaft}

Am Beginn steht dabei die Frage, wie sich das nachbarliche Zusammenleben zwischen Menschen gestaltet, die unterschiedliche Wertvorstellungen, Normenorientierungen und Lebensstile aufweisen, zum Teil über eine geringe Sprachkompetenz (in der Sprache des Aufenthaltslandes) zur gegenseitigen Kommunikation verfügen und deren Verhältnis nur allzuoft von ökonomischer und Arbeitsmarktkonkurrenz gekennzeichnet ist.

Zuwanderer sind zum Teil auch durch spezifische „fremde“ Lebensweisen und ,auffällige“ äußere Merkmale charakterisiert. Dies erleichtert den Prozess der Vorurteilsbildung und Kategorisierung seitens der „Alteingesessenen“. Selbst bei ansonsten nur schwach ausgeprägter Gruppenkohäsion innerhalb einer Nachbarschaft können sich durch den Zuzug von Migranten ablehnende Vorurteile leicht bilden. Kleinhans (1980: 109 ff.) betont, dass sich sogar in sehr heterogenen Nachbarschaften durch den Zuzug von Migranten ein deutliches Wir-Gefühl herausbilden kann. Eine hohe Homogenität der Nachbarschaft kann demnach sogar zu umso heftigeren Ausschließungstendenzen gegenüber neuen Nachbarn führen (Pettigrew 1973: 36). Je homogener die bisherige Nachbarschaft ist, desto harmlosere Konflikte reichen aus, um Ablehnung gegenüber Neuzuzüglern hervorzurufen.

Negative Reaktionen von Inländern gegenüber Migranten als Nachbarn können einerseits durch direkte Störungen hervorgerufen werden, bedürfen solcher aber nicht, um aufzubrechen, denn:

- Bereits durch die Wahrnehmbarkeit und Sichtbarkeit der Zuwanderer wird die Vertrautheit der eigenen Umwelt gestört.

- Durch den Sukzessionsprozess nimmt die Zahl inländischer Interaktionspartner im Wohnumfeld ab; Frustrationen können die Folge sein.

- Haben Immigranten eine analoge Wohnsituation wie die im Haus wohnhaften Inländer, so ist eine soziale Absetzung nach unten im Wohnbereich nicht mehr möglich; das Gerechtigkeitsempfinden kann gestört werden.

- Es kann sich direkte Konkurrenz um knappen Wohnraum oder die Benützung von Infrastruktur ergeben.

Gerade letzteres, die Nutzungskonkurrenz um knappe (hauseigene oder wohnumfeldbezogene) Infrastrukturkapazitäten, bedingt Konflikte in besonders hohem Ausmaß.

Die Wohnnachbarschaft mit ihren weniger formalisierten Kontakten bietet einen erheblichen individuellen Gestaltungsspielraum für soziale Interaktionen. Gerade im Kontext des ethnisch gemischten Wohnens kann es aber auch zu Kollisionen konträrer Verhaltensstandards kommen. Ein hochgradig dizipliniertes Wohnverhalten seitens vieler Mitteleuropäer trifft auf ein Verhalten bei nicht wenigen Migranten, das durch ein allgemein intensiveres und kommunikativeres Sozialverhalten, Erfahrungsdefizite im Umgang mit Haustechnik und mit der Anpassung an urbanes Wohnverhalten sowie manchmal durch die Unkenntnis rechtlich-abstrakter Normen im Wohnbereich 
(z.B. Hausordnungen) gekennzeichnet ist. Konfliktstiftend kann sich vor allem das höhere Maß der Außenorientierung im Wohn- und Freizeitverhalten, in der Kommunikation und in den alltäglichen Lebensabläufen seitens vieler Migranten auswirken. Nachbarn mit Migrationshintergrund sind in Stiegenhäusern, Höfen und hauseigenen Grünanlagen nach außen hin oft sichtbarer und bereits ihre Anwesenheit wird von Inländern vielfach als störend empfunden, selbst wenn unmittelbare Störungen gar nicht vorliegen (vgl. Eichener 1988: 127; Reppé \& Reiter 1997). „Einheimische und ausländische Nachbarn werden durch die aus der Gemeinsamkeit des Wohnorts resultierenden Interdependenzen in eine Figuration eingebunden, in der jede Handlung, ja, die bloße Anwesenheit spürbare Auswirkungen auf die Situation der anderen hat" (Eichener 1988: 129). Vor allem in den gründerzeitlichen Häusern mit GangWC wird ein Teil der privaten Sphäre in die Halböffentlichkeit der Stiegenhäuser verlagert. Die Grenzen zwischen Öffentlichkeit und Privatheit verschieben sich damit. Aufgezwungene Interaktionen durch die Nutzung von infrastrukturellen Einrichtungen wirken aber nicht per se kontaktfördernd im positiven Sinne (Thum 1979: 123).

Konflikte in „gemischten“ Nachbarschaften zeigen eine Frequenzsteigerung bzw. Verschärfung meist im Zusammenhang mit der steigenden Zahl/einem steigenden Anteil „fremder“ Nachbarn (Eichener 1988: 132 ff.). Mit der zahlenmäßigen Größe einer Gruppe von Zuwanderern nimmt auch deren „Auffälligkeit“ zu. Dieser Prozess ist wahrnehmungspsychologisch erklärbar. Empirische Erhebungen bestätigen, dass bereits andersartiges Verhalten, das einen Fremdkörper in der vertrauten Umwelt der Wohnumgebung darstellt, genügt, um als Störung auf Seiten der Inländer klassifiziert zu werden (vgl. Hemmer \& Leminsky 1974: 46; Friedrichs 1977: 247 ff.; Elschenbroich \& Schweitzer 1982: 11; Eichener 1988: 130).

Interethnische Nachbarschaftsbeziehungen unterliegen aber auch einer Dynamik und Veränderungen im Zeitablauf. Die bereits älteren Daten des kommunalen Mikrozensus „Leben in Köln - Umfrage 1988“ lassen den Schluss zu, dass sich der nachbarschaftliche Annäherungsprozess zwischen Deutschen und Zuwanderern in Köln zwischen 1981 und 1988 positiv entwickelt hat (Kessler \& Ross 1991: 438). Andererseits zeigen sich auch zeitliche Schwankungen in der Bereitschaft von Migranten zum Zusammenwohnen mit Inländern in der Wohnnachbarschaft (König et al. 1986). Böltken (1991: 493 ff.) ortete im Bereich der Wohnnachbarschaft ein manifestes Problem der deutschen Wohnbevölkerung vor allem mit türkischen Zuwanderern. ${ }^{16}$ Nach der Art der Kontakte untersuchte er funktionale Alltagskontakte am Arbeitsplatz und in der Nachbarschaft und expressive Beziehungen, d.h. selbstgewählte Kontakte im Freundes- und Bekanntenkreis. Türken schnitten bei ersteren nicht schlechter $\mathrm{ab}$ als die anderen Migrantengruppen sehr wohl aber bei der zweitgenannten Kon-

16 Mit $12 \%$ berichten Türken von den fünf befragten Migrantengruppen (Spanier, Italiener, Griechen, Jugoslawen, Türken) am häufigsten von „Reibereien“ mit deutschen Nachbarn (Böltken 1991: 498). 
taktkategorie. Nach Böltken ist das „normale“ Nachbarschaftsverhältnis zwischen Türken und Deutschen zwar relativ konfliktfrei, doch deutlich distanziert.

Die Unterschiede in der Kontakthäufigkeit und -intensität betreffen nach Geissler (1992: 160) auch die jüngere Generation mit türkischem Migrationshintergrund.

Von Wichtigkeit im Kontext des wohnnachbarschaftlichen Kontakt- und Konfliktgeschehens scheint der Faktor der Sichtbarkeit und das damit vielfach einhergehende Ausmaß an sozialer Distanz zu bestimmten Gruppen zu sein. Wir haben dazu auch in unsere Befragung eine Frage eingeschaltet, die wir aus dem deutschen ALLBUS übernahmen, aber im Wortlaut und bezüglich der zu bewertenden Migrantengruppen modifizierten. Die Bedeutsamkeit des Faktors der sozialen Distanz wurde bereits in zahlreichen Studien herausgearbeitet, von denen hier aber bloß einige angeführt werden sollen (Institut für Demoskopie Allensbach 1982; Just \& Mülhens 1982; Drevermann et al. 1983; Kuhlbrodt 1984; Allerbeck \& Hoag 1986; Schubarth 1990; Plasser \& Ulram 1991; Eckert \& Kissler 1992; Fischer 1992; Markefka 1995; IHS 1998).

\section{Sozialkontakte und Konflikte im Kontext der Erforschung von Nachbarschaftsbeziehungen}

\subsection{Die Kontakthypothese}

Die vorliegenden empirischen Befunde weisen zwar in keine einheitliche Richtung, sie sind aber als Rechtfertigung ausreichend, um davon auszugehen, dass die konkreten Erfahrungen, die Inländer mit Migranten in ihrem unmittelbaren Wohnumfeld machen, einen Einfluss auf die Einstellungen der betreffenden Personen gegenüber Zuwanderern allgemein bewirken können. Es ist damit also die als theoretische Ausgangshypothese in zahlreiche sozialwissenschaftliche Studien einfließende Kontakthypothese angesprochen.

Die Pionierstudie, welche viele künftige Untersuchungen bis zum heutigen Tage wesentlich beeinflusste, ist G. W. Allports „The Nature of Prejudice“ (1954, 1956). Diese bezieht sich zwar nicht primär auf nachbarschaftliche Interaktionen, deckt diese aber mit ihrem Fokus auf interethnischen Kontakten ebenfalls ab. Allport ging von einer präzisen Taxonomie von Faktoren aus, die die Resultate interethnischer Kontakte determinieren. Zu diesen Faktoren zählen (a) der quantitative Aspekt des Kontakts (z.B. Häufigkeit, Dauer, Zahl der involvierten Personen, Vielfalt), der (b) Status des Kontakts (abhängig davon, ob die Angehörigen der darin involvierten Minorität den gleichen, einen unter- oder übergeordneten Sozialstatus innehaben), die (c) Rollenaspekte des Kontakts (kompetitiver oder kooperative Sozialbeziehung, Unterordnung), (d) die soziale Atmosphäre, in welche der Sozialkontakt eingebettet ist (findet der Kontakt freiwillig oder unfreiwillig statt, ist er „,natürlich“ oder „künstlich“, typisch oder stellt er eine Ausnahmesituation dar), (e) die Persönlichkeitsmerkmale (Alter, Bildungsniveau, Vorurteilslevel) der in den Sozialkontakt involvierten Individuen 